\title{
Influence of drinking structured water to human psychophysiology
}

\begin{abstract}
Aim: The aim of the study was to study the impact of the consumption of structured water on the human body in normal environmental conditions during one month.

Materials and methods: The following techniques have been used in the study: study of body composition by bioelectric impedance; clinical and biochemical blood analysis; psychological testing; heart rate variability (HRV) method; Bio-Well GDV method. This study was an open, randomized, comparative study in which the main performance indicators were evaluated in 15 subjects - an experimental group using structured water - 1 liter per day for a month, compared to 15 subjects in the control group (using unstructured water of the same brand for a month). . Comparative tests were carried out initially and after 1 month.
\end{abstract}

Results: Both groups demonstrated an increase in the average concentration of hemoglobin in erythrocytes, which indicated an improvement in water-salt metabolism, which was due to the regular water consumption regardless of its structure. The experimental group revealed a significant reduction in total body weight due to reduction of fat mass; a significant decrease in creatinine levels, as well as an increase in glomerular filtration of the kidneys, which reflects the improvement of renal excretory function and a decrease in the level of endogenous intoxication of the body; the growth of HRV and Bio-Well GDV parameters reflecting parasympathetic activity and variability of heart rhythm and the decrease of parameters which reflect the degree of sympathetic regulation and centralization of heart rhythm. No such changes were observed in the control group, but there was a reliable negative dynamics of parameters for most of participants.

Conclusions: From the analysis of experimental data was concluded that structured water consumed 1 liter per day for a month contributes to the reduction of body fat mass, improvement of water-salt metabolism, improvement of kidney excretory function, reduction of endogenous intoxication of the body, increase of adaptive and stress-response and reserve capacity of the body even in adverse seasonal, climatic and epidemiological period. In contrast, in the control group there was a reliable negative dynamics of parameters for most of participants. The experiment was conducted during the unfavorable seasonal, climatic and epidemiological period - the period of seasonal immunodeficiency, depression and reduced efficiency. At the same time, this investigation should be considered as a pilot study, as the sample size $(n=30)$ is too small to draw any decisive conclusions. Since the study includes different age groups, sexes, and profession with that small sample size, it becomes even more complicated to draw any conclusions. We hope, that presented results stimulate development of new studies, dedicated to the influence of water to human health and wellbeing.

Keywords: structured water, health, wellness, heart rate variability, bio-well GDV
Volume 6 Issue 4 - 2019

\author{
Korotkov KG,' Churganov OA, ${ }^{2}$ Gavrilova \\ EA, ${ }^{2,3}$ Belodedova MA, ${ }^{3}$ Korotkova AK' \\ 'Saint Petersburg Research Institute of Physical Culture and \\ Sport, Russia \\ ${ }^{2}$ North-Western Medical University, Russia \\ ${ }^{3}$ Medical-sports clinic of Krasnogvardeisky district, St. \\ Petersburg, Russia
}

Correspondence: Konstantin G Korotkov, St. Petersburg Research Institute of Physical Culture and Sport, NIIFK, Ligovski prospect 56E, St. Petersburg, 19104, Russia, Tel +7-9219368394, Fax+7-8I26004II7, Email korotkov2000@gmail.com

Received: June 04, 2019 | Published: July 19, 2019

\section{Introduction}

The question of structuring water under the influence of different devices causes contradictory judgments, despite the large amount of available experimental data. ${ }^{1}$ At the same time, there are practically no publications that consider the influence of structured water on the human body in compliance with generally accepted protocols. In the present pilot study, we have set a goal to check the validity of these claims.

\section{Goals and objectives of the study}

The aim of the study was to study the impact of the consumption of structured water on the human body in normal environmental conditions during one month.

\section{Study design}

This study was an open, randomized, comparative study in which the main performance indicators were evaluated in 15 subjects - an experimental group using structured water - 1 liter per day for a month, compared to 15 subjects in the control group (using unstructured water of the same brand for a month). After the first test 30 liters of potable water were provided to the home of every participant of the study by AquaBalt Co, St. Petersburg. Comparative tests were carried out initially and after 1 month. All data collected in the study were analyzed using descriptive and dispersive statistical methods. Clinical and functional data and their changes relative to the baseline level were analyzed using the Student's T-test, Wilcoxon and MannWhitney criteria. To estimate the significance of fractions (\%) in the samples, the Fisher's Angular Transformation method was applied. 


\section{Participants of the study}

30 adults of different sexes, ages, professions, age $50+/-14$ years took part in the study. Each subject received information about the study prior to the study and signed Informed Consensus Form. Information obtained in the course of the study that identifies the identity of the subjects was kept secret and may be disclosed only within the limits established by the law. Protocol was approved by the ethic committee of the Medical-sports clinic of Krasnogvardeisky district, St. Petersburg.

\section{Criteria for inclusion and exclusion of subjects in the study}

The study included adults meeting the following criteria:

a. Absence of severe chronic medical conditions,

b. Good mental health,

c. Ability to comply with the procedures set out in the Protocol of Inquiry.

d. There was no early abandonment of the subjects from the experiment.

\section{Methods}

The following techniques have been used in the study:

a) Study of body composition by bioelectric impedance (bioimpedance meter Diamant-AIST-IRGT” by Diamant Co, Russia).

b) Clinical and Biochemical blood analysis (automatic hematological analyzer "CELL-DYN 3700" by ABBOTT and automatic biochemical analyzer "Integra 400" by Roche and kits from the same manufacturer).

c) Psychological testing.

d) Questionnaire "Feeling, activity, mood" (FAM) is intended for self-assessment of the following psychological qualities: feeling of well-being, activity, mood.

e) Heart rate variability (HRV) method. The rhythmogram was performed at rest using the Neurosoft device when analyzing 5 -minute records of heart rhythm variability. In interpreting the data, the standards for evaluating heart rhythm variability developed by the European Heart Society and the North American Society of Electrophysiology2,3 have been used.

f) Bio-Well GDV method.4 Bio-Well test was performed at rest using device by Bio-Well Co (www.bio-well.com).

\section{Water structuring}

From the theoretical principles based on quantum electrodynamics 5 follows that liquid water should be a multiphase, non-equilibrium and, therefore, the active complex system. That alone makes water a complex dynamic system with much richer behavior than that of any homogenous matter. For example, under certain conditions it should change its state in response to the weak resonance signals, and also for a long time maintain such a condition. This property is known as the "structured water. ${ }^{1,6}$ In the last decades, convincing experimental evidence has appeared, supporting the theoretical model of Italian physicists. It was shown, for example, that a sufficient condition for the emergence of a long-living organized phase in aqueous systems is the presence of the interface of liquid water with hydrophilic surfaces with which it contacts, and even the interfaces of water with air. ${ }^{7}$ The Professor of the University of Washington Gerald Pollack received this evidence. ${ }^{8}$ He has developed a simple experimental model, using which only in a few years had made a number of discoveries showing how complex is even very clean water. All these conditions promote water separation into two different substances (phases). Interaction of these phases having very different properties is the cause of many phenomena. This is closely related to the special properties of water in living matter: in particular, in the cell protoplasm. Having a long history, is the idea that water in living cells, filled with proteins, polysaccharides, and nucleic acids, must be structured due to the interaction with all these particles and so must differ in its properties from "normal" water. Since the beginning of the last century, many prominent physiologists, biochemists, chemists, and cytologists have defended this idea. They proceeded from the obvious to any unbiased scholar facts, and thus formed the basis of colloid chemistry. ${ }^{9}$ The different states of water in the cellular and extra-cellular phases prevent these two phases from mixing (and therefore, this concept is called the phase theory of protoplasm). Water in protoplasm differs in solvent capacity from the external water and the uneven distribution of substances between cell and environment should be explained - not by the presence of specific pumps and channels in a hypothetical semi-permeable membrane that separates cell from the environment - but by different distribution coefficients between the two aqueous phases. ${ }^{10-12}$ The water in erythrocytes possesses a quasi-crystalline structure, and the molecules form a complex spatial network whose loops contain hemoglobin molecules. Appropriate calculations showed, that in blood, nearly all the water occurs in the interfacial state. There are a lot of evidences that drinking structured water is beneficial for health, ${ }^{13-16}$ but not enough published research papers. This pilot study was conducted to check the validity of these claims.

For structuring water, Amezcua Bio Disc 3, an energy wellness glass product by QNet was used. This glass disc with diameter of 3.5 inch $(9 \mathrm{~cm})$ and 0.4 inch $(1 \mathrm{~cm})$ thick is made up of a special combination of natural granulated crystals, energised using their very own proprietary process technology and has a masterful geometry design that create a field of essential energy-balancing qualities and optimally balance the body's interactions with environmental stressors. The Amezcua Bio Disc 3 by QNet is similar to glass being used in solid state lasers. It transforms the light flux falling on it into quasi-coherent radiation. Water in a transparent jar placed on top of the Amezcua Bio Disc 3 becomes structured after 10 minutes and continues to increase in structure, as it has been shown by physical experiments.

\section{Results}

Due to the big amount of research parameters we are presenting only parameters demonstrating difference between initial and after moth tests both for the experimental and control groups.

\section{Bioimpedance data}

In the experimental group statistically significant decrease in the total body weight $(\mathrm{p}<0.01)$ due to the fat mass $(\mathrm{p}<0.01)$ was detected. No changes were noted in the control group. No significant differences were found in other bioimpedance parameters. However, there was an unreliable decrease in the percentage of active cell mass in the control group. 


\section{Blood analysis data}

An increase in the mean concentration of hemoglobin in erythrocyte (MCHC - mean corpuscular hemoglobin) was observed in both groups $(334 \pm 6,7$ and $341,4 \pm 7,9 \mathrm{~g} / 1$ in experimental group $(\mathrm{p}<0,001)$ vs $331,4 \pm 9,1$ and $341,9 \pm 9,2 \mathrm{~g} / 1$ in control group $(\mathrm{p}<0,001)$. The erythrocytic MCHC index shows how densely the hemoglobin molecules are concentrated in erythrocyte or how rich red blood cells are in important chromoprotein, which is in constant interaction with oxygen and carbon dioxide. Its growth may indicate an improvement in water-salt metabolism, which occurs when good water is consumed in sufficient amount. In addition, there was a significant increase in hemoglobin in the control group. The level of endogenous intoxication of the body was judged by molecules of medium mass (total cholesterol, creatinine, urea) and the enzyme gamma glutamyltransferase. Biochemical analysis data are presented in Table 1. As it follows from the table, in the experimental group there was a significant decrease in the level of creatinine and growth of the glomerular filtration of the kidneys, which reflects the improvement of the excretory function of the kidneys and a decrease in the level of endogenous intoxication. Figure 1 graphically shows the dynamics of these indicators.

Table I Biochemical Blood parameters

\begin{tabular}{lllll}
\hline Indicator & Experimental group & & \multicolumn{2}{c}{ Control group } \\
\hline & 1 test & 2 test & 1 test & 2 test \\
CHOL $(\mathrm{mmol} / \mathrm{l})$ & $4,7 \pm 0,9$ & $4,8 \pm 0,8$ & $5,9 \pm 1,2$ & $6,2 \pm 1,2$ \\
GGT (u/l) & $14,9 \pm 7$ & $13,4 \pm 5,2$ & $32,1 \pm 26,9$ & $31,1 \pm 27,8$ \\
CREA (mmol/1) & $86,5 \pm 10,6$ & $71,0 \pm 11,8 * * *$ & $90,5 \pm 15,4$ & $87 \pm 24,4$ \\
UREA (mmol/1) & $4,9 \pm 1,4$ & $4,4 \pm 11,8$ & $5,6 \pm 1$ & $5,5 \pm 1,9$ \\
CKD-EPI (ml/min) & $80 \pm 13,4$ & $100,5 \pm 14,8 * * *$ & $78,6 \pm 15$ & $84,1 \pm 20,9$
\end{tabular}

$* * * \mathrm{p}=0,001$

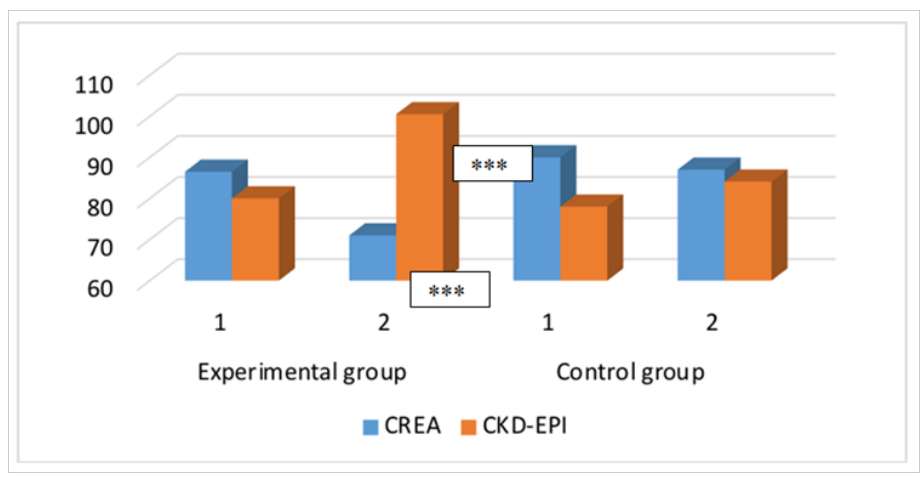

Figure I Dynamics of CREA ( $\mathrm{mmol} / \mathrm{l})$ and CKD-EPI $(\mathrm{ml} / \mathrm{min})$ during the experiment (I and 2 tests).

$* * * \mathrm{p}=0,001$.

\section{Psychological status data}

Despite a clear trend of improvement in health, activity and mood in the experimental group and a decrease in health and mood in the control group, there was no significant difference between the first and second test. This may be explained as all participants were apparently healthy people having no serious health issues (Figure 2).

\section{HRV data}

The rhythmogram was evaluated by 5 -minute recording in rest. Dynamics of median parameters of heart rhythm variability in two groups is presented in Table 2. As it follows from the table, the experimental group was characterized by the growth of parameters reflecting parasympathetic activity and variability of heart rhythm and the decrease of parameters which reflect the degree of sympathetic regulation and centralization of heart rhythm. Significant changes were noted in RMSSD (19\%), HF (26\%), VLF (24\%), LF/HF (18\%) indexes, which indicated growth of economy of respiratory and cardiovascular systems functioning, growth of adaptive and energetic potential of the organism, as well as recovery after loads.

In the control group, inverse changes in heart rhythm variability were noted, indicating increased activity of the sympato-andrenal system. Reliable changes were revealed in respect of almost all indicators, which reflected the centralization of the heart rhythm, reduction of adaptation of the organism and its reserve capacity in the adverse epidemiological, climatic and seasonal period. Figure 3 shows the dynamics of rhythmogram parameters in two groups of subjects, the increase of which reflects the growth of adaptation, regulation and reserves of the body.

As shown in the figure, the experimental group was distinguished by the positive dynamics of indicators reflecting the growth of adaptive abilities, reserve possibilities and vital energy of the organism. In contrast, the control group showed negative dynamics of these indicators. Figure 4 shows the dynamics of rhythmogram indices in two groups of subjects, the increase of which reflects the unfavorable tendency of the organism adaptation and its reserve possibilities decrease. 


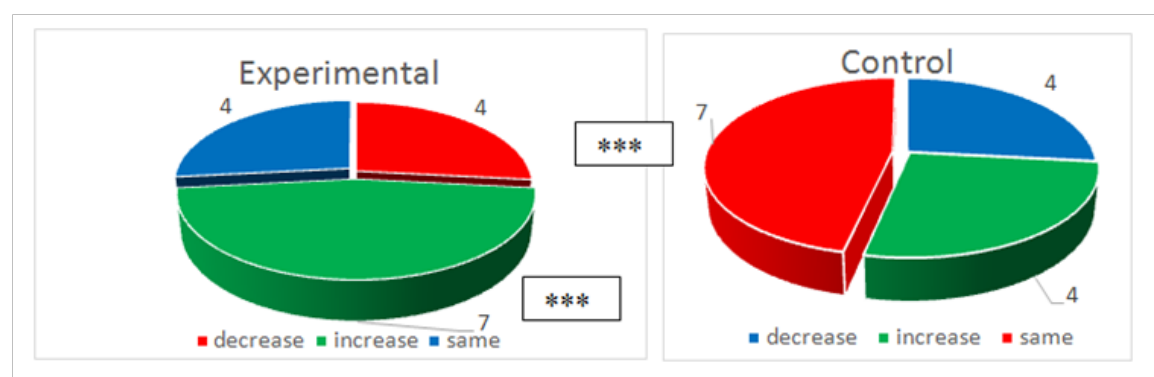

Figure 2 Change of Stress level in experimental and control groups due to self-assessment.

Table 2 Dynamics of median of indicators HRV and percentage of changes in the experimental and control group

\begin{tabular}{|c|c|c|c|c|c|c|}
\hline \multirow[t]{2}{*}{ Indicator } & \multicolumn{2}{|c|}{$\begin{array}{l}\text { Experimental group } \\
\text { (mediana) }\end{array}$} & \multirow[t]{2}{*}{ \%changes } & \multicolumn{2}{|c|}{$\begin{array}{l}\text { Control group } \\
\text { (mediana) }\end{array}$} & \multirow[t]{2}{*}{ \%changes } \\
\hline & I test & 2 test & & I test & 2 test & \\
\hline $\mathrm{RR}$ (msec) & 891,0 & 910,6 & 2,20 & 880,9 & 884,1 & 0,36 \\
\hline RMSSD (msec) & 34,6 & $4 I, 3$ & $19 *$ & 35,9 & 28,1 & $-21,73^{*}$ \\
\hline LF/HF (ratio) & 1,62 & 1,33 & $-18 *$ & 1,20 & 1,33 & 10,83 \\
\hline Mo (msec) & 850,0 & 900,1 & 5,89 & 850,1 & 850,1 & 0,00 \\
\hline TP $\left(\mathrm{msec}^{2}\right)$ & 2016,4 & 2039,0 & 1,12 & 1315,9 & 1594,5 & $21,17^{*}$ \\
\hline $\operatorname{VLF}\left(\mathrm{msec}^{2}\right)$ & 810,9 & 614,8 & $-24 * *$ & 767,4 & 931,8 & $21,42^{*}$ \\
\hline IC (ratio) & 3,14 & 2,96 & $-5,73$ & 2,76 & 4,00 & $44,93 * *$ \\
\hline HF norm & 38,2 & 44,6 & 16,75 & 61,1 & 42,9 & $-29,79 *$ \\
\hline $\mathrm{HF}\left(\mathrm{msec}^{2}\right)$ & 430,2 & 539,8 & $26 * *$ & 997,1 & 349,3 & $-64,97 * *$ \\
\hline SI (ratio) & 98,2 & 88,4 & $-9,98$ & 84,7 & $132, \mid$ & $55,96 * *$ \\
\hline SDNN (msec) & 48,0 & 52,7 & 9,79 & 60,3 & 47,0 & $-22,06$ \\
\hline LF norm (\%) & 61,8 & 57,1 & $-7,61$ & 38,9 & 57,1 & $46,79 * *$ \\
\hline Amo (\%) & 39,1 & 41,3 & 5,63 & 35,6 & 46,1 & $29,49 *$ \\
\hline LF $\left(\mathrm{msec}^{2}\right)$ & 758,2 & 644,2 & $-15,04$ & 635,7 & 528,3 & $-16,89$ \\
\hline
\end{tabular}

${ }^{*} \mathrm{p}<0,05,{ }^{*} \mathrm{p}<0,01$.

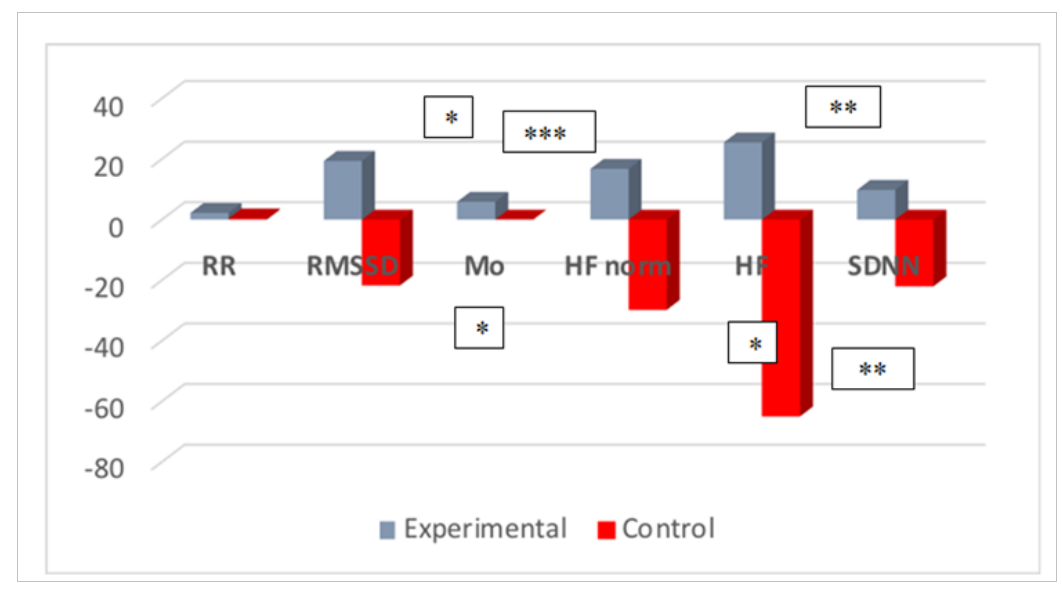

Figure 3 Percentage of change in rhythmogram indicators, the increase in which reflects the increase in adaptation and reserve capacity of an organism. $*_{\mathrm{p}}<0,05,{ }^{*} \mathrm{p}<0,0$ I. 


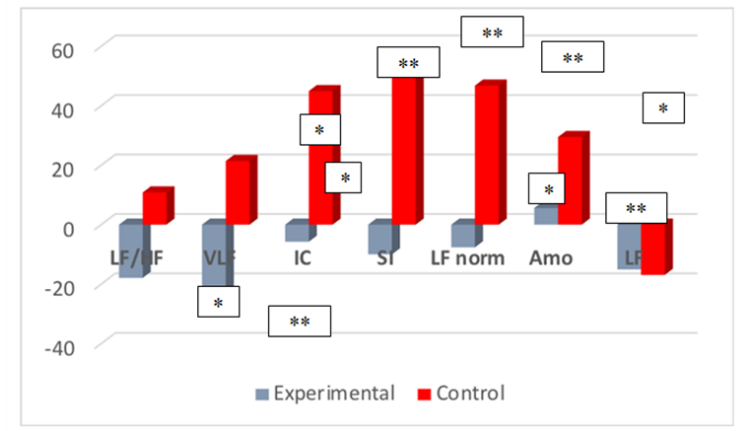

Figure 4 Percentage of change in rhythmogram parameters, the increase of which reflects the decrease in adaptation of the organism and its reserve capacity.

$* \mathrm{p}<0,05, * * \mathrm{p}<0,01$.

As we see from this figure, there is a clear opposite dynamics of indicators reflecting the decrease in adaptation of the organism in the control group, which may be explained by the effect of the adverse epidemiological, climatic and seasonal period - and the decrease of indicators in the experimental group due to the protective effect of Amezcua Bio Disc 3 water. Figure 5 reflects the dynamics of the spectral parameters of the heart rhythm of the two groups. The figures clearly demonstrate the growth of parasympathetic regulation in the experimental group and central regulation in control group. It also reflects the above described tendencies of adaptation potential in the groups.

\section{Bio-well GDV data}

For Control group no difference was found between two measurements. For Experimental group changes for the following parameters was found (Figures 6-8): Stress Index; Organs Balance; Balance L. As we see, in experimental group for 9 people Stress index decreased, for 5 people it increased staying in the optimal zone, and for 1 person it was the same. In control group For 5 people Stress index increased, for 3 people decreased, while for 7 people stayed practically the same.

As we see for all the people Organs balance improved in experimental group and decreased in control group. This index correlates with SNS/PNS balance, and its increase signifies improvement of the ANS balance.

As we see for 10 people in experimental group Balance L improved, while for 5 people it was the same and very high. In control group it decreased for 7 people, increased for 2, and was the same for 5 people. It is important to note that this index is correlated with sympathetic activity of the autonomic nervous system, and its increase in experimental group signifies decrease of sympathetic activity and increase of organs balance. For people with high balance it did not change, while for people with low balance it increased. This is reflection of growth of adaptive and energetic potential of the organism, as well as recovery after loads. In control group for 7 participants we see the opposite effect. For Area parameter it was no difference between experimental and control groups.

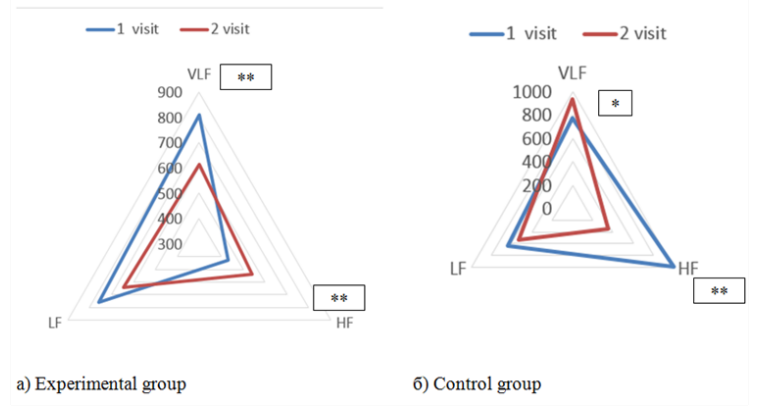

Figure 5 Dynamics of spectral parameters of heart rhythm $\left(\mathrm{msec}^{2}\right)$ in experimental (a) and control (b) groups. ${ }^{*} \mathrm{p}<0,05,{ }^{*} \mathrm{p}<0,01$.

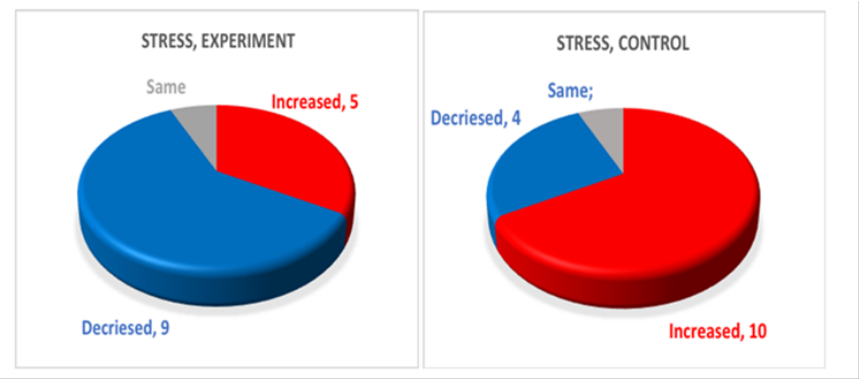

Figure 6 Change of Stress Index for 15 people from experimental and control groups after a month. 


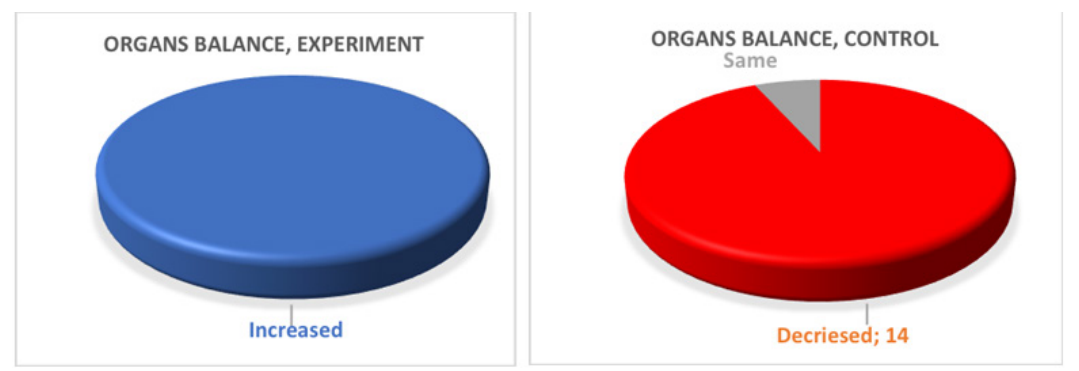

Figure 7 Change of Organs balance Index for 15 people from experimental and control groups after a month.
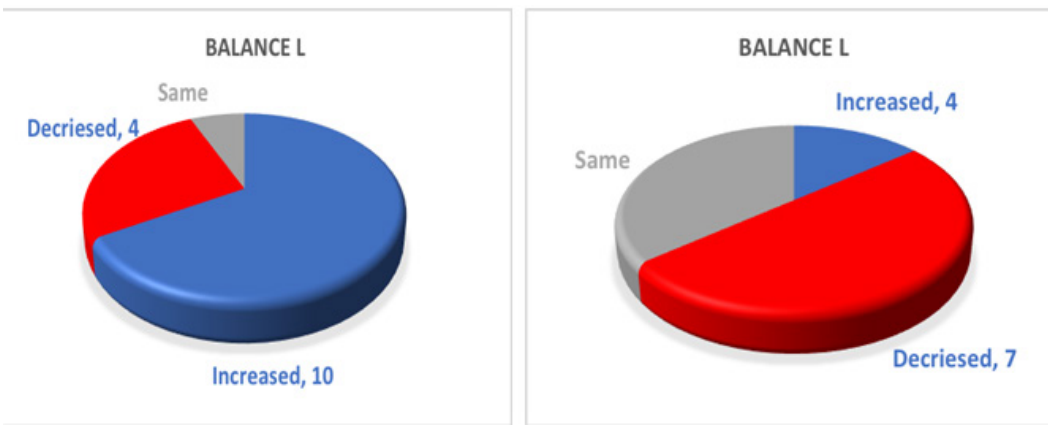

Figure 8 Balance L Index for I5 people from experimental and control groups initially and a month after.

\section{Discussion and conclusions}

From the above presented data, we can make the following conclusions:

I. The study is devoted to the influence of 30-day consumption of water structured with Amezcua Bio Disc 3 on the composition of the body and blood, the nervous and cardiovascular systems, cleansing the body of endotoxins, energy and psychological status compared to the control group that consumes unstructured water of the same brand.

II. 22 women and 8 men aged between 36 and 62 years, apparently healthy, made up two groups of subjects: 15 persons in the experimental (using structured water) and 15 persons in the control group (using unstructured water of the same brand).

III. The participants before and after 30 days of water consumption in the dose of 1 liter per day underwent a clinical survey, body composition study, clinical and biochemical blood analysis, study of the psychological status, rhythmocardiography and Bio-Well GRV analysis.

\section{Main results}

a. Both groups demonstrated an increase in the average concentration of hemoglobin in erythrocytes, which indicated an improvement in water-salt metabolism, which was due to the regular water consumption regardless of its structure.

b. The experimental group revealed a significant reduction in total body weight due to reduction of fat mass. No such changes were observed in the control group.

c. The experimental group showed a significant decrease in creatinine levels, as well as an increase in glomerular filtration of the kidneys, which reflects the improvement of renal excretory function and a decrease in the level of endogenous intoxication of the body. No such changes were observed in the control group.

d. The experimental group was characterized by the growth of HRV parameters reflecting parasympathetic activity and variability of heart rhythm and the decrease of parameters which reflect the degree of sympathetic regulation and centralization of heart rhythm. Significant changes were noted in RMSSD (19\%), HF (26\%), VLF (24\%), LF/HF (18\%) indexes, which indicated growth of economy of respiratory and cardiovascular systems functioning, growth of adaptive and energetic potential of the organism, as well as recovery after loads. In contrast, in the control group there was a reliable negative dynamics of these parameters.

e. The experimental group was characterized by the growth of BioWell GDV parameters reflecting decrease of stress level for most of participants, as well as decrease of sympathetic activity and increase of organs balance. This is reflection of growth of adaptive and energetic potential of the organism, as well as recovery after loads.

f. In contrast, in the control group there was a reliable negative dynamics of Bio-Well GDV parameters for most of participants. The experiment was conducted during the unfavorable seasonal, climatic and epidemiological period - the period of seasonal immunodeficiency, depression and reduced efficiency.

g. Thus, it can be concluded that structured with the help of Amezcua Bio Disk3 water consumed 1 liter per day for a month contributes to the reduction of body fat mass, improvement of water-salt metabolism, improvement of kidney excretory function, reduction of the endogenous intoxication of the body, increase of adaptive and stress-response and reserve capacity of the body even in the adverse seasonal, climatic and epidemiological period. 


\section{Limitations of the study}

This investigation should be considered as a pilot study, as the sample size $(\mathrm{n}=30)$ is too small to draw any decisive conclusions. Since the study includes different age groups, sexes, and profession with that small sample size, it becomes even more complicated to draw any conclusions. We hope, that presented results stimulate development of a new studies, dedicated to the influence of water to human health and wellbeing.

\section{Acknowledgements}

None.

\section{Conflicts of interest}

The author declares there are no conflicts of interest.

\section{References}

1. Voeikov V, Korotkov K. The Emerging Science of Water. 2017.

2. Heart rate variability. Standards of measurement, physiological interpretation, and clinical use. Task Force of the European Society of Cardiology and the North American Society of Pacing and Electrophysiology. Circulation. 1996;17:354-381.

3. Task Force of the European Society of Cardiology and the North American Society of Pacing and Electrophysiology. Heart rate variability. Standarts of Measurement. Physiological interpretation and clinical use. Circulation. 1996;93:1043-1065.
4. Korotkov KG. The Energy of Health. Amazon.com Publishing. 2018.

5. Del Giudice E. Preparata G, Vitiello G. Water as a Free Electric Dipole Laser. Phys Rev Lett. 1988;61:1085-1088.

6. Clark GNI, Cappa CD, Smith JD, et al. The structure of ambient water. Molecular Phys. 2010;108:1415-1433.

7. Tokushima T, Harada Y, Takahashi O, et al. High resolution X-ray emission spectroscopy of liquid water: The observation of two structural motifs. Chem Phys Lett. 2008;460:387-400.

8. Chen CS, Chung WJ, Hsu IC, et al. Force field measurements within the exclusion zone of water. J Biol Phys.2012;38:113-120.

9. Pollack GH. The Fourth Phase of Water. Seattle, Ebner \& Sons; 2013.

10. Henry M. The state of water in living systems: from the liquid to the jellyfish. Cell Mol Biol (Noisy-le-grand). 2005;51:677-702.

11. Nasonov DN. Protoplasm reactions. Moscow; 1959.

12. Ling Gilbert. The Physical State of Water in Living Cell and Model Systems. Annals of the New York Academy of Sciences. 2006;125(2):401417.

13. Ho L, Thas O, Echelpoel WV, et al. A Practical Protocol for the Experimental Design of Comparative Studies on Water Treatment. Water. 2019;11(1):162.

14. https://draxe.com/nutrition/structured-water/

15. https://www.alkalinewaterplus.com/doctors-experts/

16. http://www.crystalblueent.com/testimonials--results.html 